\title{
Barreiras e facilitadores da adesão a um programa de educação em diabetes: a visão do usuário
}

\author{
Adherence barriers and facilitators to a diabetes education program: \\ the user's point of view
}

\author{
Guilherme Falcão Mendes', Alexandre Luiz Gonçalves de Rezende', Jane Dullius', Júlia Aparecida Devidé Nogueira
}

\section{Resumo}

Este estudo objetivou identificar barreiras e facilitadores da adesão a um programa para o tratamento da Diabetes Mellitus tipo 2 (DM2). O Programa Doce Desafio (PDD) é contínuo, de base anual; regular, oferecido desde 2001; multidisciplinar, com exercícios físicos supervisionados, alimentação, autocuidado, uso de medicamentos e aspectos psicossociais; multiprofissional, com profissionais da farmácia, nutrição, educação física, medicina e enfermagem; multiestratégico, com palestras, debates, jogos, oficinas, discussões e práticas de exercício físico supervisionado; comunitário, incluindo a atenção básica; intersetorial, entre Universidade e Serviço; e realiza sessões de 120 minutos duas vezes por semana, em três locais do Distrito Federal. A pesquisa foi qualitativa, exploratória e baseada na técnica "roda de conversa". Todos os 132 frequentadores do programa foram convidados e 55 sujeitos aceitaram participar da pesquisa. Cada unidade de atendimento sediou um encontro mediado pelo pesquisador, que utilizou roteiro semiestruturado para estabelecer um diálogo sobre as barreiras e facilitadores da adesão ao PDD. Todas as falas foram gravadas, transcritas e submetidas à análise de conteúdo. Das falas emergiram seis facilitadores à adesão: motivação para aprender; incentivo familiar; interação afetiva; melhoria da saúde e qualidade de vida; prazer da atividade física; e orientação profissional. E, quatro barreiras: desgaste no preenchimento de formulários; complicações de saúde; dificuldades financeiras; e problemas familiares. Educação multidisciplinar, exercícios físicos orientados e acompanhamento humanizado foram diferenciais do PDD em relação a outras vivências no tratamento da DM2. Falta avançar na capacitação profissional e sensibilização dos participantes para que as avaliações clínicas se tornem menos exaustivas e mais acessíveis.

\section{Palavras-chave}

Educação; Exercício; Adesão do paciente; Programas de saúde.

\begin{abstract}
This study aims to identify barriers and facilitators of adherence to a program for the treatment of type 2 diabetes mellitus (DM2). The Sweet Challenge Program (PDD) is continuous, in annual basis; regular, offered since 2001; multidisciplinary, with supervised exercise, nutrition, self-care, use of medication and psychosocial aspects; multiprofessional, with pharmacy, nutrition, physical education, medicine and nursing professionals; multistrategic, with lectures, debates, games, workshops, discussions, and supervised exercise practices; community-based, including primary care; intersectoral, among University and Service; and offers 120 minutes sessions twice a week, in three places of the Federal District. The research was qualitative, exploratory and based on "conversation circle" technic. All 132 program attendees were invited and 55 accepted to participate. Each local hosted a meeting mediated by the researcher, using semi-structured script, to establish a dialogue about barriers and facilitators of adherence to PDD. All speeches were recorded, transcribed and subjected to content analysis. From speech emerged six facilitators to adherence: motivation to learn; family encouragement; affective interaction; bealth and quality of life improvement, physical activity enjoyment, and professional guidance. And, four barriers: form fillings; bealth complications, financial difficulties, and family problems. Multidisciplinary education, guided physical exercises and bumanized follow-up were $P D D$ 's differential in relation to other experiences in the treatment of DM2. There is need to progress in professional training and participants sensitization so that clinical evaluations become less exhausting and more accessible.
\end{abstract}

\section{Keywords}

Education; Exercise; Patient compliance; Health program.

\section{Introdução}

Diabetes Mellitus (DM) é um grupo de doenças metabólicas caracterizadas por um estado de hiperglice- mia sustentada ${ }^{1}$. O Brasil é o quarto país no mundo com maior número de casos de DM: 14,3 milhões de adultos, além dos 3,2 milhões de casos não-diagnosticados 2 . Do total de casos de DM, 90 a 95\% são 
diabetes tipo 2 (DM2), uma doença associada à fatores genéticos, obesidade e estilo de vida, particularmente inatividade física e alimentação inadequada ${ }^{2}$. A DM2 apresenta elevados custos pessoais, sociais e econômicos para o indivíduo, a família e a sociedade acarretados pela medicação constante, complicações à saúde, medicalização e hospitalização, absenteísmo, aposentadoria precoce, invalidez e morte prematura ${ }^{2-4}$. Os gastos do setor saúde com a DM no Brasil foram da ordem de 22 bilhões de dólares americanos em $2015^{2}$.

Dada a extensão e a gravidade desta realidade, além da promoção da saúde e da prevenção de doenças, é essencial promover o controle e o tratamento da DM2 como estratégia de saúde pública ${ }^{5}$ ponderando, inclusive, seu custo-benefí$\mathrm{cio}^{4}$. Considerando a etiologia da DM2, seu tratamento exige cuidados de saúde multidisciplinares, contínuos e permanentes, integrando alimentação saudável, atividade física, autocontrole, autocuidado, uso de medicamentos e suporte psicossocial $^{5,6}$. A Sociedade Brasileira de Diabetes ratifica que a adoção de um estilo de vida saudável, caracterizado pela prática regular de atividade física e alimentação saudável, é praticamente duas vezes mais eficaz e eficiente que o tratamento farmacológico no controle da $\mathrm{DM} 2^{6}$.

Neste sentido, as políticas públicas de saúde no Brasil apontam programas multidisciplinares de promoção da saúde como estratégias do Sistema Único de Saúde (SUS) para garantir melhor expectativa e qualidade de vida aos portadores de DM2 $2^{5,7,8}$. O modelo de atenção desenvolvido a partir da Estratégia Saúde da Família deve promover ações na atenção básica com foco na prevenção, promoção e recuperação da saúde de forma integral e contínua ${ }^{5,78}$ para evitar os custos gerados pelo tratamento em estágios mais avançados da doença ${ }^{3-5}$.

No entanto, a efetividade do controle da DM2 baseada na mudança dos hábitos de vida e nos cuidados permanentes contrasta com as exigências físicas e psicológicas desse tipo de abordagem, frequentemente resultando em baixa adesão ao tratamento ${ }^{9,10}$. Para que seja possível alcançar resultados positivos no enfrentamento da DM2 é importante facilitar o acesso aos serviços oferecidos na atenção básica, tais como dispensação de medicamentos, terapias e programas interventivos ${ }^{5,6}$, mas também, estabelecer um plano terapêutico que considere a adesão ao tratamento, aqui entendida como a habilidade do portador em seguir as prescrições e recomendações a ele propostas, entendendo os benefícios que o tratamento pode lhe trazer ${ }^{10}$. A capacidade de mobilizar o sujeito para que ele seja também responsável por sua própria saúde é um dos desafios do modelo de atenção proposto pelo SUS $5,7,8$.

Considerando a magnitude dos problemas causados pela DM2 e as diretrizes das políticas em saúde no Brasil, é fundamental a realização de estudos que contribuam para aumentar a efetividade das ações de prevenção, promoção e manutenção da saúde na comunidade ${ }^{10-12}$; e que contemplem os fatores relacionados à adesão, como aspectos a serem previstos no planejamento de ações e na operacionalização dos programas ${ }^{5,7-12}$. No intuito de contribuir para o melhor entendimento da adesão, aspecto fundamental para o sucesso terapêutico ${ }^{10}$, e favorecer o desenvolvimento de programas que contemplem o cuidado integral e participativo no modelo de atenção à saúde ${ }^{5}$, o presente estudo busca identificar barreiras e facilitadores que afetam a adesão de indivíduos com DM2 à um programa educativo com ênfase no exercício físico - o Programa Doce Desafio (PDD) - desenvolvido de acordo com as recomendações de referência para o tratamento da DM2 $2^{5,6}$. 


\section{Métodos}

O PDD é um modelo de atenção multidisciplinar ao portador de DM2 desenvolvido pela Universidade de Brasília em parceria com a Estratégia de Saúde da Família desde 2001. O objetivo do PDD é melhorar a qualidade de vida, a autonomia e o controle metabólico através de uma série de ações educativas que contemplam a realização de exercícios físicos supervisionados por estagiários do curso de educação física, a alimentação, o autocuidado, o uso de medicamentos e os aspectos psicossociais. Todas as sessões contam com monitoramento e coleta de dados da situação de saúde, tais como glicose pré e pós exercício físico. As atividades são desenvolvidas por profissionais e estudantes da área da saúde, oferecidas duas vezes por semana por cerca de 120 minutos cada sessão.

O ingresso e a participação no PDD se dá de forma espontânea e gratuita e os convites para participar são feitos por anúncios na mídia local (TV, rádio, jornal e web). Os critérios de inclusão no programa são: ter idade entre 18 e 70 anos de idade, ser diagnosticado com DM2 conforme os padrões da Sociedade Brasileira de Diabetes $^{6}$, e ter assinado o Termo de Consentimento Livre e Esclarecido. O presente estudo foi aprovado pelo Comitê de Ética em Pesquisa da Fundação de Ensino e Pesquisa em Ciências da Saúde do DF (nº 020/2009) e a pesquisa foi conduzida dentro dos padrões éticos exigidos pela Resolução n 196/96 do Ministério da Saúde.

A população do estudo foi constituída por todos os participantes do PDD no ano de 2009 ( $n=132)$. Neste ano o programa foi desenvolvido em três locais distintos: um centro comunitário no Plano Piloto ( $\mathrm{PP}, \mathrm{n}=53$ ), região de nível socioeconômico médio-alto ${ }^{13}$; e em duas Unidades Básicas de Saúde (UBS) localizadas nas cidades satélites de Samambaia (Sa, n= 47) e Sobradinho (So, n= 32), regiões de baixo nível socioeconômico do Distrito Federal ${ }^{13}$. A adesão de cada sujeito ao programa foi classificada de acordo com sua frequência nos 66 encontros realizados pelo PDD no ano de 2009, sendo dividida em: baixa (frequência menor que $33,3 \%$ ); moderada (frequência de 33,4 a 66,6\%); e alta (frequência acima de 66,7\%).

O estudo é de natureza qualitativa, com caráter exploratório e baseado na técnica da "roda de conversa", também conhecida como discussão em grupo ${ }^{14}$. Todos os frequentadores do programa foram convidados a participar de uma "roda de conversa" visando identificar as barreiras e facilitadores que interferem na adesão ao PDD. Cada unidade de atendimento (PP, Sa e So) sediou um único encontro agendado previamente em dia e horário de atividade regular do PDD. A técnica de "roda de conversa" foi conduzida de forma similar nos três grupos pelo mesmo pesquisador, que utilizou um roteiro de perguntas semiestruturado (Quadro 1) para iniciar, guiar e dinamizar o diálogo.

QUADRO 1 - Questões norteadoras do roteiro semiestruturado de acordo com as categorias de facilitadores e barreiras à adesão no Programa Doce Desafio. Brasília, 2009.

\begin{tabular}{|c|c|}
\hline Categorias & Questões do roteiro semi-estruturado \\
\hline \multirow{4}{*}{ Facilitadores } & 1. Que aspectos facilitaram (motivaram) seu ingresso no PDD? \\
\hline & 2. Que aspectos facilitam (motivam) sua permanência no PDD ? \\
\hline & $\begin{array}{l}\text { 3. Qual a principal diferença entre o trabalho desenvolvido no PDD e outros programas de } \\
\text { atendimento ao diabético? }\end{array}$ \\
\hline & 4. Para você, o que tem mais importância no atendimento do PDD? \\
\hline \multirow{2}{*}{ Barreiras } & $\begin{array}{l}\text { 5. Em sua opinião, quais atividades do programa mais dificultam (desestimulam) sua } \\
\text { participação e frequência no PDD? }\end{array}$ \\
\hline & $\begin{array}{l}\text { 6. Quais os fatores relacionados à sua vida que dificultam (desestimulam) sua participação e } \\
\text { frequência no PDD? }\end{array}$ \\
\hline
\end{tabular}

PDD; Programa Doce Desafio. 
A roda de conversa foi realizada em uma sala reservada aos participantes do estudo para evitar interferências externas e com as cadeiras dispostas em círculo para facilitar a comunicação ${ }^{14}$. Informações sobre a garantia de sigilo; de que cada um poderia expressar livremente sua opinião, fornecendo mais de uma ou nenhuma resposta para cada questão; e de que qualquer resposta, inclusive as destoantes do senso comum, eram relevantes; foram enfatizadas em cada grupo. A seguir o pesquisador leu, uma de cada vez, as questões do roteiro semiestruturado e estimulou que as pessoas interessadas expressassem sua opinião. Ao longo das respostas, o pesquisador solicitava esclarecimentos adicionais, evitava a dispersão e controlava o tempo para que todos tivessem oportunidade de falar. Todo o processo foi gravado em áudio digital e contou com anotações adicionais por parte de dois relatores da pesquisa. Cada roda de conversa teve duração aproximada de uma hora.

Todas as falas foram transcritas literalmente e submetidas à análise de conteúdo $^{14}$ com o auxílio do software de análise textual Nvivo 2008. A priori, partiu-se de duas categorias: barreiras e facilitadores que, a partir da análise textual, foram divididas em subcategorias temáticas. $\mathrm{O}$ uso do software auxiliou a identificação das principais subcategorias emergentes que facilitam ou dificultam a adesão de indivíduos com DM2 ao PDD. Falas que ilustraram as categorias de respostas mais frequentes foram destacadas para subsidiar a discussão.

\section{Resultados}

Dados gerais do PDD em 2009 registraram 132 participantes (73,0\% mulheres) com idade média e desvio padrão $( \pm \mathrm{DP})$ de $62,0 \pm 10,1$ anos, tempo de diagnóstico de DM2 de 8,0 $\pm 6,2$ anos, e 23,0\% em insulinoterapia. Destes, 53,0\% apresentaram baixa adesão, 31,8\% moderada adesão e 15,2\% alta adesão ao programa. Quando estratificados por polos de atendimento, uma diferença entre os participantes foi o nível de escolaridade, sendo que no PP a maioria (83,0\%) reportou ter, no mínimo, o ensino médio, e nas cidades satélites (Sa e So) 81,0\% reportaram ter, no máximo, o nível fundamental completo (dados não apresentados em tabela).

Dentre os frequentadores do PDD, 55 pessoas (71,0\% mulheres) participaram da "roda de conversa" (15 participantes no PP, 22 em So e 18 em Sa). A idade média ( \pm DP) foi de 60,7 $\pm 10,6$ anos, o tempo de diagnóstico da DM2 foi de 8,3 $\pm 6,5$ anos, e 24,0\% reportaram estar em insulinoterapia; dados similares à população do programa. Não obstante, os participantes da "roda de conversa" apresentaram níveis mais elevados de adesão ao programa: $23,6 \%$ apresentaram baixa adesão, $50,9 \%$ moderada e $25,5 \%$ alta adesão ao PDD.

A partir da análise de conteúdo das falas dos participantes emergiram seis subcategorias de facilitadores à adesão no PDD (motivação para aprender; incentivo da família; interação afetiva; melhoria da saúde e qualidade de vida; prazer da atividade física; e orientação profissional) e quatro subcategorias de barreiras (desgaste no preenchimento de formulários; complicações de saúde; dificuldades financeiras; e problemas familiares).

De modo a permitir a discussão mais aprofundada de cada fator influenciador da adesão, são apresentadas a seguir a prevalência das categorias de respostas obtidas para facilitadores (Tabela 1) e barreiras (Tabela 2) separadas por polos de atendimento. Como cada participante pode expressar mais de uma resposta a cada pergunta, as prevalências totais para cada polo podem ser superiores a 100\%. Em adição, algumas falas representativas de cada contexto foram transcritas para subsidiar a argumentação subsequente. 
TABELA 1 - Distribuição por frequência das respostas dos usuários a partir da análise temática sobre os facilitadores da adesão ao Programa Doce Desafio (PDD) separadas por polos de atendimento. Brasília, 2009.

\begin{tabular}{|c|c|c|c|c|}
\hline \multirow{2}{*}{$\begin{array}{l}\text { Questão } \\
\text { 1. O que te motivou a ingressar no PDD? }\end{array}$} & \multicolumn{3}{|c|}{ Polos de atendimento } & \multirow[b]{2}{*}{ Total $(n=49$} \\
\hline & $P P(n=11)$ & $\mathrm{Sa}(\mathrm{n}=14)$ & So $(n=25)$ & \\
\hline Necessidade de informação em DM & 0,0 & 85,7 & 32,1 & 42,0 \\
\hline Encaminhamento familiar & 37,5 & 0,0 & 14,3 & 14,0 \\
\hline Encaminhamento profissional & 0,0 & 14,3 & 14,3 & 12,0 \\
\hline Piora da condição diabética & 25,0 & 0,0 & 14,3 & 12,0 \\
\hline Melhorar a qualidade de vida & 25,0 & 0,0 & 14,3 & 12,0 \\
\hline Apoio do grupo no PDD & 12,5 & 0,0 & 10,7 & 8,0 \\
\hline 2. O que te motiva a permanecer no PDD? & $P P(n=17)$ & $\mathrm{Sa}(\mathrm{n}=18)$ & So $(n=22)$ & Total $(n=57)$ \\
\hline A qualidade do trabalho do PDD & 50,0 & 0,0 & 66,7 & 37,0 \\
\hline Melhora da saúde/ qualidade de vida/ bem estar & 0,0 & 38,9 & 33,3 & 28,3 \\
\hline Educação/ conhecimentos/ conscientização obtidos & 0,0 & 38,9 & 0,0 & 15,2 \\
\hline Convivência com os diabéticos & 40,0 & 0,0 & 0,0 & 8,7 \\
\hline Convivência com os profissionais & 0,0 & 16,7 & 0,0 & 6,5 \\
\hline Os exercícios físicos promovidos & 20,0 & 5,6 & 0,0 & 6,5 \\
\hline $\begin{array}{l}\text { 3. Qual a principal diferença entre o trabalho } \\
\text { desenvolvido no PDD e outros programas de } \\
\text { atendimento ao diabético? }\end{array}$ & $P P(n=15)$ & $\mathrm{Sa}(\mathrm{n}=20)$ & So $(n=22)$ & Total $(n=57)$ \\
\hline Reconhece diferenças no trabalho do PDD & 55,6 & 100,0 & 71,4 & 79,2 \\
\hline Não conhece outros programas para diabéticos & 33,3 & 0,0 & 19,0 & 14,6 \\
\hline Comodidade e proximidade da moradia & 0,0 & 11,1 & 4,8 & 6,3 \\
\hline $\begin{array}{l}\text { 4. Para você, o que tem mais importância no } \\
\text { atendimento do PDD? }\end{array}$ & $P P(n=13)$ & $\mathrm{Sa}(\mathrm{n}=18)$ & So $(n=22)$ & Total $(n=53)$ \\
\hline As atividades físicas e seus benefícios & 55,6 & 41,2 & 38,1 & 42,6 \\
\hline A companhia dos profissionais/monitores & 0,0 & 47,1 & 52,4 & 40,4 \\
\hline As atividades educativas & 22,2 & 11,8 & 9,5 & 12,8 \\
\hline
\end{tabular}

DM, diabetes mellitus; PP, Plano Piloto; Sa, Samambaia; So, Sobradinho; n, número total de respostas para cada questão.

Os relatos apontam que os facilitadores para o ingresso no PDD foram a motivação para aprender e a necessidade de obter informação sobre DM e/ou o incentivo e a preocupação da família em buscar um atendimento adequado.

"Tenho diabetes há 25 anos e minha familia viu o programa no jornal[...] Semestre passado me incentivaram a vir [...]" (PP2).

"[...] Precisava aprender um pouco o que é diabetes e também aceitar essa doença que eu não conhecia” (Sa5).

"[...] tenho que conhecer a diabetes, tenho pouco tempo de diagnóstico, e eu não sabia sobre a doença, ingressei para aprender, e aprendi muito” (So18).

Em relação aos facilitadores para permanência no PDD, 37,0\% responderam que foi a qualidade do trabalho do PDD (caracterizada pelo detalhamento das informações e a humanização do atendimento); e 28,3\% responderam a melhora da saúde, qualidade de vida ou bem estar (devido a adequação do controle glicêmico, pelo prazer da atividade física, pelo enfoque multidisciplinar e pelo contato social e afetivo), como observado nas falas. 
"[... o tipo de informação que recebemos, não recebo em lugar nenhum [...]”. (PP13).

"O que me motivou foi o saber, as explicação que vocês dão pra nós, a recomendação de alimentação que foi passada, [...] eu não era muito ligada nisso" (Sa18).

"Tô aqui pela ginástica, e as medições da pressão e da diabetes. O que me motivou a estar aqui também foram vocês, vocês são muito gentis. [...] é melhor do que ficar assistindo televisão em casa, né” (So4).

Sobre a principal diferença do trabalho do PDD, comparado a outros programas, $79,2 \%$ reconheceu alguma diferença, expressando o fato de ter, por exemplo: explicações detalhadas, diálogo, ensinamentos, compreensão, carinho, acolhimento, humanização, multidisciplinariedade, entre outros. E 14,6\% respondeu que não conhece outros programas para diabéticos, como pode ser observado nas falas a seguir:

"Não conheço outros programas, mas o PDD é bumanizado [...]" (PP8).

"Pra mim tem grande diferença, a qui a gente é acompanhado por uma equipe profissional de muitas repartições (multiprofissional), e lá (no centro de saúde) é só um. [...] A gente fica que nem cego sem saber o que fazer" (Sa10).

"Atendimento só conheço do PDD, porque médico é médico, [...] mas aqui vocês fazem algo a mais" (So7).

Sobre o aspecto mais importante do trabalho desenvolvido no PDD, a maioria respondeu que são: os exercícios físicos supervisionados e seus benefícios; e a companhia dos profissionais/monitores como expresso nas falas:

"Não adianta exercícios físicos sem instrução, nem aprender sem prática [...]" (PP9).

"Atividade física é muito bom, [...] fundamental, e com a assistência de vocês fica ainda melhor" (Sa1).

"A companhia dos monitores é mais importante, pois sem vocês não estaríamos praticando atividades físicas" (So12).

TABELA 2 - Distribuição por frequência das respostas dos usuários a partir da análise temática sobre barreiras à adesão ao Programa Doce Desafio (PDD) separadas por polos de atendimento. Brasília, 2009.

\begin{tabular}{lcccc}
\hline \multicolumn{1}{c}{ Questão } & \multicolumn{3}{c}{ Polos de atendimento } & \\
\hline $\begin{array}{l}\text { 5. Em sua opinião, quais atividades do PDD que mais te } \\
\text { desestimulam? }\end{array}$ & $\begin{array}{c}P P \\
(n=15)\end{array}$ & $\begin{array}{c}\text { Sa } \\
(n=18)\end{array}$ & $\begin{array}{c}\text { So } \\
(n=22)\end{array}$ & $\begin{array}{c}\text { TOTAL } \\
(n=55)\end{array}$ \\
\hline As fichas de inscrição e encerramento & 53,8 & 69,2 & 90,5 & 74,5 \\
\hline Aferição da glicemia & 15,4 & 23,1 & 4,8 & 12,8 \\
\hline O preenchimento da ficha diária & 15,4 & 7,7 & 4,8 & 8,5 \\
\hline $\begin{array}{l}\text { 6. Para você, quais são os fatores relacionados à sua } \\
\text { vida que dificultam a sua participação no PDD? }\end{array}$ & $\begin{array}{c}P P \\
(n=14)\end{array}$ & $\begin{array}{c}\text { Sa } \\
(n=18)\end{array}$ & $\begin{array}{c}\text { So } \\
(n=22)\end{array}$ & $\begin{array}{c}\text { TOTAL } \\
(n=54)\end{array}$ \\
\hline Não relataram dificuldade & 54,5 & 27,8 & 18,2 & 29,4 \\
\hline Situação familiar & 0,0 & 22,2 & 36,4 & 23,5 \\
\hline Situação física / doença & 9,1 & 22,2 & 31,8 & 23,5 \\
\hline Situação financeira / trabalho & 27,3 & 22,2 & 9,1 & 17,6 \\
\hline
\end{tabular}

DM, diabetes mellitus; PP, Plano Piloto; Sa, Samambaia; So, Sobradinho; n, número total de respostas para cada questão.

Sobre as barreiras na rotina do PDD, $83,0 \%$ comentaram sobre o desgaste com o preenchimento dos formulários de avaliação do programa, seja no momento da inscrição, do encerramento ou no atendimento cotidiano: 
"Às vezes eu esqueço das coisas, é difícil pra mim (relatar as informações solicitadas nas fichas) [...]" (Sa14).

"[...] porque (nas fichas) tem coisa que a gente nem sabe, é muita coisa pra pensar" (So21).

"Tem aquela última ficha né [...] é muita coisa que nem lembro, é muito cansativo [...]" (So5).

Sobre as barreiras na vida diária que dificultam a participação e a frequência no PDD, 29,4\% respondeu espontaneamente que não tinha dificuldade, o que é compreensível pela composição da amostra, que reúne pessoas com maior adesão, como também, pela dificuldade para relatar em grupo, suas dificuldades pessoais. Dentre os que apontaram algumas dificuldades, as principais foram adoecimento, situação familiar e situação financeira como observado nas falas a seguir:

"O trabalho divide meu tempo [...] às vezes chego atrasada" (PP7).

"Tem os problemas de saúde né [...] e os gastos com a saúde [...] às vezes fica difícil vir (ao PDD)" (Sa3)

"Às vezes não tenho dinheiro para a passagem de ônibus [... e e tenho medo de deixar meus filhos sozinhos em casa" (Sa10).

"Às vezes tenho que faltar por causa das complicações (da DM2) [...] infecção, hemodiálise [...]” (So11).

\section{Discussão}

A presente pesquisa identificou conjuntos de fatores facilitadores e barreiras à adesão ao PDD. Dentre os facilitadores se destacaram o conhecimento, a família, o acolhimento, a saúde, o prazer e a orientação; e dentre as barreiras se destacaram a avaliação, a saúde, a renda e a família. As subcategorias "saúde" e "família" possuem uma característica ambivalente, pois tanto foram citadas como facilitadores, dentro de um determinado contexto, como barreiras, quando estão em outro contexto.

Estes resultados corroboram com os achados de outros estudos que destacam que o ser humano raramente atua (ou deixa de atuar) com base num único motivo; sua motivação funciona como um conjunto de forças que lhe levam a agir, influenciado por suas experiências e outros fatores externos ${ }^{15,16}$.

As subcategorias facilitadoras identificadas no presente estudo condizem com o modelo de autorregulação do comportamento ${ }^{17}$, que defende a coexistência de dois processos ativos influenciando os comportamentos de autocuidado na doença: a cognição, que corresponde à interpretação objetiva da ameaça de doença e busca por informações; e a emoção, reação subjetiva à ameaça, que busca apoio social ${ }^{17}$.

Neste sentido, cabe refletir sobre o conceito de risco, hegemônico no discurso da saúde, que parte da suposição que a adesão aos comportamentos saudáveis é determinada racionalmente pela vulnerabilidade das pessoas às ameaças à sua saúde, e ignora que o processo emotivo é interativo e paralelo à cognição ${ }^{15}$. O aspecto emocional fica claro quando os usuários do PDD apontam a convivência social com profissionais e colegas do programa como importante fator de facilitação à adesão, e utilizam palavras como gentileza, diálogo, acolhimento, compreensão, carinho e humanização para definir o diferencial do PDD frente a outros atendimentos. A formação de uma rede de apoio social (família, amigos e profissionais da saúde) é primordial para a conscientização e adoção das mudanças necessárias e estão associadas com a adesão ao tratamento em DM2 ${ }^{16,18}$.

Em adição, as falas que reportam a qualidade do trabalho e as informações prestadas pelo PDD como principal facilitador para permanecer no programa 
permitem ponderar se as estratégias tradicionais dos serviços de saúde - uma vez que os participantes do PDD também são usuários dos sistemas de saúde - tem atendido a esfera cognitiva de busca por informações sobre a doença. Estudos apontam que a falta de conhecimento e capacitação acerca da DM e a pouca integração entre os profissionais está relacionada à baixa adesão ao tratamento ${ }^{19,20}$. O modelo predominante de atendimento na atenção básica ainda se baseia na triagem e retornos periódicos centrados na figura do médico ${ }^{11,21,22}$. A escassez de equipes multiprofissionais e o incipiente trabalho multidisciplinar e em rede na Estratégia Saúde da Família tornam as ações deficitárias e limitadas, e prejudicam a integralidade do cuidado ${ }^{11,20}$.

Outro aspecto facilitador da adesão ao PDD foram os exercícios físicos supervisionados e regulares. Estudos indicam que a motivação de adultos com DM2 para participar de exercícios pode estar relacionada tanto à melhoras progressivas no quadro clínico (perda de peso e diminuição ou supressão do uso de medicamentos) e à sensação de bem-estar, quanto à socialização promovida por estas atividades (a supervisão profissional e o encontro com pares) ${ }^{23,24}$, principalmente em comunidades mais carentes ${ }^{4,11,18}$. Em adição, alguns autores apontam a difícil manutenção da prática regular de exercícios fora de grupos ou classes que tenham um objetivo em comum ${ }^{24,25}$.

Corroborando com os achados do presente estudo, outros autores apontam fatores intrínsecos (prontidão para a modificação do estilo de vida e aceitação da doença) e extrínsecos (apoio social, familiar e profissional) que podem melhorar a adesão a programas de educação em DM2 ${ }^{16,18}$. O envolvimento familiar contribui para adesão e autogestão da DM2, principalmente fora dos espaços supervisionados e a convivência com grupos e pessoas próximas faz com que os encontros frequentes se tornem uma rotina prazerosa, proporcionando melhor adesão ao tratamento e controle metabólico ${ }^{9,16,18}$. Neste sentido, a prática de exercícios físicos como meio para a educação em saúde tem grande potencial para promover a interação social e aumentar a sensação de bem-estar quando comparado a outros componentes do tratamento (modificação dos hábitos alimentares, uso de medicamentos e automonitoração da glicemia), podendo aumentar a adesão ao programa ${ }^{24}$.

Ao analisar as barreiras à adesão nota-se que as avaliações a que os participantes são submetidos no programa são vistas como desestimulantes. Devido ao caráter científico e multidisciplinar do PDD, vários questionários são preenchidos no ingresso e no decorrer do programa, em adição às fichas de anamnese da UBS. Como diversas perguntas exigem memória e habilidade de relatar, há maior dificuldade entre os participantes mais idosos e com menor escolaridade, aspecto relatado também em outros estudos ${ }^{26,27}$.

Outras barreiras relatadas foram problemas de saúde associados à DM2 e a outras comorbidades, bem como demandas familiares. É comum pessoas com DM2 se sentirem sobrecarregadas ou frustradas no contexto biopsicossocial da doença e a exaustão resultante do estresse pode ocasionar a renúncia ou falta de adesão, quando a pessoa opta por largar tudo, inclusive o tratamento ${ }^{18,28}$. Tais barreiras foram mais frequentemente referidas pelos participantes com menor nível educacional e econômico, indicando que esses são importantes determinantes sociais da saúde $e^{7,11}$.

Fatores externos como a dificuldade de acesso, inclusive financeira, ao tratamento foram destacados nas falas de participantes de menor nível socioeconômico neste e em outros estudos ${ }^{4,12}$. A proximidade da moradia foi apontado como facilitador e a falta de dinheiro para a passagem como barreira à adesão ao PDD em participantes das localidades de Sa e So. Cabe aqui destacar a dicotomia da 
subcategoria "situação financeira/trabalho" pois, a despeito dos termos aparecerem juntos nas falas dos sujeitos, é possível supor que as limitações financeiras estejam, de certa forma, inversamente relacionadas às demandas de trabalho.

A adesão a tratamentos continuados representa um desafio aos programas e sistemas de saúde ${ }^{5,16}$ e uma dificuldade a ser vencida pelo sujeito ${ }^{12}$. A baixa adesão terapêutica ocasiona prejuízos à qualidade de vida pessoal, familiar, social e sobrecarrega os sistemas de saúde ${ }^{6,16}$. Assim, o presente estudo buscou discutir a relevância da estrutura organizacional, da proposta andragógica, dos fatores individuais e dos determinantes sociais no processo de adesão ao tratamento, de forma a subsidiar a reflexão crítica e favorecer o desenvolvimento de programas, ambientes e estratégias que propiciem o cuidado integral e participativo no modelo de atenção à saúde e de controle da DM2 $2^{5-8}$.

No caso do PDD, o programa é desenvolvido de acordo com as políticas de saúde que estimulam programas de educação em DM2 na atenção básica ${ }^{7,8}$, e de estudos que apontam ações multiprofissionais a partir da Estratégia Saúde da Família como custo efetivas ${ }^{3,4,6}$ e favoráveis ao apoio social no controle da DM2 ${ }^{11,16,20}$. O programa é ofertado de forma contínua e regular desde 2001; com atendimento multidisciplinar por profissionais da farmácia, nutrição, educação física, medicina e enfermagem; e desenvolvimento de ações multiestratégicas de educação e exercícios físicos supervisionados (alongamentos, aeróbicos e de resistência muscular). O programa é intersetorial, numa parceria entre a Universidade de Brasília e a Secretaria de Saúde do Distrito Federal, e atende a comunidade inclusive nas UBS.

Não obstante, a adesão é um fenômeno complexo ${ }^{16}$. Este e outro estudo ${ }^{29}$ apontam que, mesmo quando os usuários foram atendidos por equipe multiprofissional numa abordagem ampliada de qualidade de vida, boa parte não aderiu ao tratamento. A adesão implica uma atitude ativa, com envolvimento voluntário e colaborativo do portador e do profissional de saúde, num processo conjunto que conduz ao reconhecimento da importância das ações prescritas e visa a mudança de comportamento ${ }^{15,19}$. Programas terapêuticos que envolvam mudanças do estilo de vida precisam considerar a motivação dos pacientes, familiares, cuidadores e pessoas relacionadas ${ }^{9}$, e promover mecanismos negociados entre todos para minimizar o abandono do tratamento e o agravamento da enfermidade ${ }^{29}$.

Ao solicitar que os participantes do PDD relatassem as barreiras e facilitadores à adesão, buscou-se favorecer a autonomia e o empoderamento através do amadurecimento do papel ativo do usuário ${ }^{8}$ e o olhar sobre sua própria condição de demandante de auxílio/orientação/cuidado, ampliando a oportunidade de reflexão do indivíduo, em conjunto com os profissionais de saúde, sobre as possibilidades de soluções para os problemas e os benefícios da modificação de seus hábitos ${ }^{15,28}$.

Em suma, os participantes do estudo destacaram o conhecimento, a família, o acolhimento, a saúde, o prazer e a orientação como facilitadores da adesão ao PDD. As informações disponibilizadas, o apoio social (dos familiares, dos profissionais de saúde e dos colegas do grupo) e as preocupações com sua saúde e qualidade de vida foram fundamentais para motivar o ingresso e a adesão ao programa. O acompanhamento humanizado, contínuo e multidisciplinar e os exercícios físicos foram aspectos importantes e diferenciais do PDD em relação a outras vivências no tratamento da DM2. No entanto, mesmo sendo um programa de educação desenvolvido por uma equipe multidisciplinar em parceria com a Estratégia Saúde da Família, o PDD apresentou barreiras à adesão, principalmente relacionadas à avaliação e coleta de informações, além de aspectos relacionados à dificuldades com a saúde, a renda e a família. 
A despeito dos participantes da "roda de conversa" apresentarem um perfil semelhante à totalidade dos participantes do PDD em relação ao sexo, idade e tempo de diagnóstico da DM2, é relevante destacar que a amostra apresentou níveis mais elevados de adesão ao programa. Este viés de auto-seleção era esperado uma vez que a amostra foi composta espontaneamente pelos interessados que estavam presentes no dia da realização da pesquisa (marcada em horário de atividade regular do PDD). Além disso, a amostra teve maior representatividade dos sujeitos com menor nível educacional e socioeconômico, não sendo, nesses aspectos, representativa do grupo.

Outras limitações do estudo também estão relacionadas aos aspectos metodológicos da pesquisa. Por sua característica qualitativa, exploratória e com amostragem intencional, a pesquisa não teve intenção de obter representatividade populacional. É possível que pessoas com menor adesão ao programa enfrentem outras barreiras para manter a regularidade da participação e que, devido às diferenças no nível socioeconômico, essas barreiras sejam menos relacionadas às questões financeiras.

Conhecer e fortalecer os pontos positivos e ajustar os pontos negativos é fundamental para favorecer a adesão ao tratamento, ampliando os benefícios à saúde e qualidade de vida dos usuários. Nesse sentido, o PDD vem trabalhando para sensibilizar os participantes a respeito da relevância das avaliações e anamneses, ações imprescindíveis para o acompanhamento clínico; bem como para capacitar os profissionais de forma que esses processos não sejam tão exaustivos nem passem a sensação de incompreensão àqueles com dificuldades em responder. O processo avaliativo deve ser realizado periodicamente para otimizar o planejamento terapêutico e a tomada de decisões buscando reduzir continuamente as barreiras e motivar o grupo para superar os desafios e ampliar cada vez mais a adesão às práticas promotoras da saúde ${ }^{30}$.

\section{Agradecimentos}

Agradecemos a participação da Secretaria Estadual de Saúde do Distrito Federal; dos usuários dos Centros de Saúde $\mathrm{n}^{\circ} 2$ de Sobradinho, $\mathrm{n}^{\circ} 1$ de Samambaia, e do Centro Olímpico da Universidade de Brasília; e dos bolsistas de iniciação científica e voluntários que atuaram no programa. Pelo apoio financeiro ao projeto agradecemos à Fundação de Ensino e Pesquisa em Ciências da Saúde (FEPECS-DF), ao Fundo de Apoio à Pesquisa (FAP-DF), e à Coordenação de Aperfeiçoamento de Pessoal de Nível Superior (CAPES).

\section{Contribuição dos autores}

G.F. Mendes (0000-0003-4985-6996), A.L.G. Rezende (0000-0003-0515-4319), J. Dullius (0000-0003-3170-2016) e J.A.D. Nogueira (0000-0002-0318-1973) contribuíram igualmente para a redação e revisão do manuscrito, e aprovaram a versão final a ser publicada. J.A.D. Nogueira (0000-0002-0318-1973) e G.F. Mendes (0000-0003-4985-6996) foram os autores que conceberam o artigo e o adequaram às normas da revista; G.F. Mendes (0000-0003-4985-6996) foi o pesquisador à frente da coleta de dados e busca de referencias bibliográficas; A.L.G. Rezende (0000-0003-0515-4319) contribuiu exponencialmente com a análise e interpretação das informações; e J. Dullius (0000-0003-3170-2016) é a responsável por manter o projeto em andamento.

\section{Conflito de interesse}

Os autores declaram não haver conflitos de interesse. 


\section{Referências}

1. American Diabetes Association. Standards of medical care in diabetes, 2014. Diabetes Care. 2014;37(Supl 1):14-80.

2. International Diabetes Federation. 7th Diabetes Atlas. Executive summary. Belgium, Brussels. Update 2015. [citado 2017 mar 08]. Disponível em: https://www.idf.org/ diabetesatlas

3. Jacobs-van der Bruggen MA, Bos G, Bemelmans WJ, Hoogenveen RT, Vijgen SM, Baan CA. Lifestyle interventions are cost-effective in people with different levels of diabetes risk: results from a modeling study. Diabetes Care. 2007;30(1):128-34.

4. Bahia LR, Araujo DV, Schaan BD, Dib SA, Negrato CA, Leão MP, et al. The costs of type 2 diabetes mellitus outpatient care in the Brazilian public health system. Value Health. 2011;14(5):137-40.

5. Brasil. Ministério da Saúde. Cadernos de Atenção Básica - n. ${ }^{\circ} 16$-Série A. Diabetes Mellitus. Normas e Manuais Técnicos. Secretaria de Atenção à Saúde. Departamento de Atenção Básica. Brasília: DF, 2006. [citado 2017 mar 08]. Disponível em: http://bvsms. saude.gov.br/bvs/publicacoes/diabetes_mellitus.PDF

6. Sociedade Brasileira de Diabetes. Diretrizes da SBD: 2013-2014. São Paulo: AC Farmacêutica Ltda, 2014. [citado 2017 mar 08]. Disponível em: http://www.sgc.goias. gov.br/upload/arquivos/2014-05/diretrizes-sbd-2014.pdf

7. Brasil. Portaria n ${ }^{\circ} 2.488$, de 21 de outubro de 2011. Aprova a Política Nacional de Atenção Básica, estabelecendo a revisão de diretrizes e normas para a organização da Atenção Básica, para a Estratégia Saúde da Família (ESF) e o Programa de Agentes Comunitários de Saúde (PACS). Diário Oficial da República Federativa do Brasil, Brasília, n.204, p.55, 24 out. 2011. [citado 2017 mar 08]. Disponível em: http://bvsms.saude.gov.br/bvs/ saudelegis/gm/2011/prt2488_21_10_2011.html

8. Brasil. Ministério da Saúde. Secretaria de Vigilância em Saúde, Secretaria de Atenção à Saúde. Política Nacional de Promoção da Saúde. Brasília: Ministério da Saúde; 2010. [citado 2017 mar 08]. Disponível em: http://bvsms.saude.gov.br/bvs/publicacoes/ politica_nacional_promocao_saude_3ed.pdf

9. Santos ECB, Zanetti ML, Otero LM, Santos MA. O cuidado sob a ótica do paciente diabético e de seu principal cuidador. Rev Latino-am Enfermagem. 2005;13(3):397-406.

10. Lerman I. Adherence to treatment: a key for avoiding long-term complications of diabetes. Arch Med Res. 2005;36(3):300-6.

11. Guidoni CM, Olivera CMX, Freitas O, Pereira LRL. Assistência ao diabetes no Sistema Único de Saúde: análise do modelo atual. Braz J Pharm Sci. 2009;45(1):37-48.

12. Assunção MCF, Santos IS, Costa JS. Avaliação do processo da atenção médica: adequação de pacientes com diabetes mellitus. Cad Saúde Pública. 2002;18(1):205-11.

13. Codeplan. Pesquisa distrital por amostra de domicílios - PDAD/2015. Brasília, 2016. [citado 2017 mar 08]. Disponível em: http://www.codeplan.df.gov.br/areas-tematicas/ pesquisas-socioeconomicas/319-pdad-2015.html

14. Flick U. Introdução à pesquisa qualitativa. Porto Alegre: Artmed, 2009.

15. Deci EL, Ryan RM. The "what" and "why" of goal pursuits: human needs and the selfdetermination of behavior. Psychol Inq. 2000;11(4):227-68.

16. Costa JDA, Balga RSM, Alfenas RDCG, Cotta RMM. Promoção da saúde e diabetes: discutindo a adesão e a motivação de indivíduos diabéticos participantes de programas de saúde. Ciênc Saúde Coletiva. 2011;16(3):2001-9.

17. Leventhal H, Benyamini, Y, Brownlee S, Diefenbach M, Leventhal EA, Patrick-Miller L, et al. Illness representation: theoretical foundations. In: Petrie k, Weinman J. Perceptions of health and illness: current research \& applications. Singapore: Harwood Academic Publishers; 1997. p.19-46.

18. Villas Boas LCG, Foss MC, Freitas MCF, Pace, AE. Relação entre apoio social, adesão aos tratamentos e controle metabólico de pessoas com diabetes mellitus. Rev Latino-Am Enfermagem. 2012;20(1):1-8.

19. Apóstolo JLA, Viveiros CSC, Nunes HIR, Domingues HRF. Incerteza na doença e motivação para o tratamento em diabéticos tipo 2. Rev Latino-am Enfermagem. 2007;15(4):575-82.

20. Loch-Neckel G, Seemann G, Eidt HB, Rabuske MM, Crepaldi MA. Desafios para a ação interdisciplinar na atenção básica: implicações relativas à composição das equipes de saúde da família. Ciênc Saúde Coletiva. 2009;14:1463-72. 
21. Assunção TS, Ursine PGS. Estudo de fatores associados à adesão ao tratamento não farmacológico em portadores de diabetes mellitus assistidos pelo Programa Saúde da Família, Ventosa, Belo Horizonte. Ciênc Saúde Coletiva. 2008;13(2):2189-97.

22. Araújo RB, Santos I, Cavaleti MA, Costa JSD, Béria JU. Avaliação do cuidado prestado a pacientes diabéticos em nível primário. Rev Saúde Pública. 1999;33(2):24-32.

23. Casey D, De Civita M, Dasgupta K. Education and Psychological Aspects Understanding physical activity facilitators and barriers during and following a supervised exercise program in Type 2 diabetes: a qualitative study. Diabet Med. 2010;27:79-84

24. Avery L, Flynn D, Van Wersch A, Sniehotta FF, Trenell MI. Changing physical activity behavior in type 2 diabetes: a systematic review and meta-analysis of behavioral interventions. Diabetes Care. 2012;35(12):2681-9.

25. Korkiakangas EE, Alahuhta MA, Laitinen JH. Barriers to regular exercise among adults at high risk or diagnosed with type 2 diabetes: a systematic review. Health Promot Int. 2009;24(4):416-27.

26. Paulo DLV, Yassuda MS. Queixas de memória de idosos e sua relação com escolaridade, desempenho cognitivo e sintomas de depressão e ansiedade. Rev Psiquiatr Clín. 2010;37(1):23-6.

27. Moreira FD, Dullius J, Karnikowski MGO, Novaes MRCG. Atividades Educativas na adesão ao tratamento com idosos diabéticos: complicadores e facilitadores do processo. Rev Portuguesa Diabetes. 2009;4(3):111-9.

28. Polonsky WH. Emotional and quality-of-life aspects of diabetes management. Curr Diab Rep. 2002;2(2):153-9.

29. Leite SAO, Costa PABC, Guse C, Dorociaki JG, Silveira MC, Teodorovicz R, et al. Enfoque multidisciplinar ao paciente diabético: avaliação do impacto do Staged Diabetes Management em um sistema de saúde privado. Arq Bras Endocrinol Metab. 2001;45(5):481-6.

30. Kluding PM, Singh R, Goetz J, Rucker J, Bracciano S, Curry N. Feasibility and Effectiveness of a Pilot Health Promotion Program for Adults with Type 2 Diabetes: Lessons Learned. Diabetes Educ. 2010;36(4):595-602.

\section{Endereço para \\ Correspondência \\ Júlia A.D. Nogueira \\ julianogueira@unb.br}

Faculdade de Educação Física da

Universidade de Brasília, Campus

Universitário Darcy Ribeiro, Asa Norte,

Brasília-DF. CEP: 70910-970.
Recebido

Revisado

$30 / 11 / 2015$

$19 / 10 / 2016$

Aprovado $\quad 11 / 03 / 2017$ 